\title{
Electronic noise of warm electrons in semiconductors from first principles
}

\author{
Alexander Y. Choi $\odot,{ }^{*}$ Peishi S. Cheng $\odot,{ }^{*}$ Benjamin Hatanpää $\odot$, and Austin J. Minnich ${ }^{\dagger}$ \\ Division of Engineering and Applied Science, California Institute of Technology, Pasadena, California 91125, USA
}

(Received 23 September 2020; revised 25 January 2021; accepted 1 March 2021; published 6 April 2021)

\begin{abstract}
The ab initio theory of low-field electronic transport properties such as carrier mobility in semiconductors is well-established. However, an equivalent treatment of electronic fluctuations about a nonequilibrium steady state, which are readily probed experimentally, remains less explored. Here, we report a first-principles theory of electronic noise for warm electrons in semiconductors. In contrast with typical numerical methods used for electronic noise, no adjustable parameters are required in the present formalism, with the electronic band structure and scattering rates calculated from first principles. We demonstrate the utility of our approach by applying it to GaAs and show that spectral features in $\mathrm{AC}$ transport properties and noise originate from the disparate time scales of momentum and energy relaxation, despite the dominance of optical phonon scattering. Our formalism enables a parameter-free approach to probe the microscopic transport processes that give rise to electronic noise in semiconductors.
\end{abstract}

DOI: 10.1103/PhysRevMaterials.5.044603

\section{INTRODUCTION}

Charge transport in semiconductors is a topic of fundamental and practical interest with a well-established theoretical foundation $[1,2]$. In many cases, a sufficient understanding of the relevant physics at both low and high fields can be achieved using the Boltzmann equation with semiempirical scattering rates [3-6]. In other cases, a more precise description of the electronic transitions induced by phonons and other perturbations is required. Such a description is now possible owing to advances in electronic structure codes that enable the ab initio computation of the transition matrix elements given by Fermi's golden rule performed in conjunction with the numerical solution of the Boltzmann equation describing carrier dynamics [7-10]. While method development is ongoing, these calculations are now routine for various semiconductors including Si [11-13], GaAs [14-16], phosphorene [7], and others [17-21].

In contrast, an equivalent treatment of fluctuations from a nonequilibrium steady-state is lacking, despite the experimental accessibility of electronic noise $[22,23]$ and its importance in applications [24]. At equilibrium, the Nyquist relation, or more generally the fluctuation-dissipation theorem, relates the electrical conductivity to the spectral noise power [25-27]. Outside of equilibrium, the theorem no longer applies and the spectral noise power must be computed with another approach.

The theoretical description of fluctuations about a nonequilibrium steady-state has a long history. In 1935, Leontovich used kinetic theory to examine velocity fluctuations of a nonequilibrium gas [28]. Around 20 years later, Wannier established the definition of a diffusion coefficient for transport about a nonequilibrium steady state [29]. Hashitsume con-

\footnotetext{
*These authors contributed equally to this work.

†Corresponding author: aminnich@ caltech.edu
}

sidered a microscopic description of occupancy fluctuations about a steady distribution using the Fokker-Planck equation with a random source term [30]. In analogy with earlier works on fluctuational Maxwell equations, Kadomotsev introduced Langevin sources into the Boltzmann equation [31]. Shortly thereafter, Price derived that for spatially homogeneous fluctuations, a fluctuation-diffusion relation links Wannier's diffusion coefficient to the spectral density of current fluctuations even outside of equilibrium [32]. For this reason, the nonequilibrium noise at frequencies small compared to scattering rates is known as diffusion noise. In the same year, Lax formulated a general kinetic theory of fluctuations for a Markovian system [33]. Throughout the 1960s, Gantsevich and co-workers applied Lax's kinetic theory to dilute gases for which the evolution of the one particle distribution function is governed by the linear Boltzmann equation [34]. Their technique, termed the "method of moments," demonstrated how to compute the spectral density of current fluctuations using only the solutions of the linear Boltzmann equation. Concurrently with Gantsevich, starting from Kadomotsev's Boltzmann-Langevin equation, Kogan and Shul'man developed a Langevin treatment of the current density fluctuations [35]. Lax, van Vliet, and Kogan and Shul'man independently confirmed that the method of moments and the Langevin approach are equivalent [36-38].

As computational resources became increasingly available, numerical implementations of the methods described above permitted computations of electronic noise for both warm $\left(\Delta T / T_{0} \ll 1\right)$ and hot $\left(\Delta T / T_{0} \sim 1\right)$ electrons, where $\Delta T$ is the steady-state temperature rise of the electrons and $T_{0}$ is the lattice temperature. Due to the lack of knowledge of the precise transition rates between electronic states, these studies employed simplified band structures and parameterized models for scattering such as deformation potential theory for acoustic phonon scattering [39,40]. For example, Stanton and Wilkins obtained the Green's function of the Boltzmann equation under the single-mode relaxation time 
approximation, demonstrating qualitative agreement with experiment in GaAs for one [41] and two [42] valleys. Numerous Monte Carlo simulations reported calculations of current spectral densities in Si [39,43-47], GaAs [44,48,49], and other semiconductors [50-53]. These works employed various approximations such as Debye acoustic phonons, dispersionless optical phonons, and spherical approximations for electron conduction bands. With empirical knowledge of band structure parameters such as effective mass and approximate relaxation times, reasonable agreement with experiments was reported [39,43-48,50,54,55]. More recently, these methods have been extended to heterostructures and have provided insight into the design of low-noise devices [56-58]. While studies with parameterized models can provide an adequate description of the physics of interest in certain cases, they are not predictive and are restricted to materials for which empirical models of the dominant scattering mechanisms are available. It is therefore natural to consider how advances in the ab initio calculation of mean transport quantities [8,9] can be applied to the nonequilibrium steady state.

Here, we present an ab initio theory of electronic noise for warm electrons in nondegenerate semiconductors. The formalism provides the spectral noise power and AC transport quantities without any adjustable parameters. Using the method, we show that the anisotropy and spectral features of the noise power in GaAs can be explained by the disparate timescales of momentum and energy exchange with phonons, even though scattering is dominated by the inelastic polar optical phonon scattering mechanism. The formalism is easily extendable to other semiconductors of technological interest such as $\mathrm{InP}, \mathrm{Si}$, and $\mathrm{Ge}$. Our method provides a parameter-free view of the microscopic transport processes responsible for electronic fluctuations in semiconductors and will advance fundamental studies of carrier transport and applications of low noise semiconductor devices.

\section{THEORY}

\section{A. Steady-state transport}

We begin by reviewing the ab initio treatment of steadystate transport using the Boltzmann equation to set the notation. Consider a nondegenerate, spatially homogeneous electron gas subject to an external electric field $\mathcal{E}$. The system is governed by the following Boltzmann equation:

$$
\frac{\partial f_{m \mathbf{k}}}{\partial t}+\sum_{\gamma} \frac{e \mathcal{E}_{\gamma}}{\hbar} \frac{\partial f_{m \mathbf{k}}}{\partial_{k_{\gamma}}}=\mathcal{I}\left[f_{m \mathbf{k}}\right] .
$$

Here, $f_{m \mathbf{k}}$ is the distribution function that describes the occupancy of the electron state with wave vector $\mathbf{k}$ and band index $m, e$ is the fundamental charge, $\hbar$ is the reduced Planck constant, and $\gamma=x, y, z$ indexes the crystal axes.

The collision integral, $\mathcal{I}$, describes the scattering rates between electronic Bloch states $m \mathbf{k}$ and $m^{\prime} \mathbf{k}^{\prime}$. In general, the collision integral is a nonlinear functional of the distribution function given by Fermi's golden rule [1]. In the steady case, the transient term vanishes, and we denote the solution of the resulting equation as $f_{m \mathbf{k}}^{s}$.

In many problems, a good approximation is that the Boltzmann equation can be linearized about an equilibrium distribution as $f_{m \mathbf{k}}^{s} \equiv f_{m \mathbf{k}}^{0}+\Delta f_{m \mathbf{k}}$, where $\Delta f_{m \mathbf{k}}$ is the change in occupation due to the electric field $\mathcal{E}$ relative to the equilibrium distribution $f_{m \mathbf{k}}^{0}$. Under the nondegenerate assumption, $f_{m \mathbf{k}}^{0}$ is well approximated by the Maxwell-Boltzmann distribution. With this substitution and retaining only terms linear in $\Delta f_{m \mathbf{k}}$, the Boltzmann equation becomes [3]

$$
\sum_{\gamma}\left[\frac{e \mathcal{E}_{\gamma}}{\hbar} \frac{\partial \Delta f_{m \mathbf{k}}}{\partial_{k_{\gamma}}}\right]+\sum_{m^{\prime} \mathbf{k}^{\prime}} \Theta_{m \mathbf{k} m^{\prime} \mathbf{k}^{\prime}} \Delta f_{m^{\prime} \mathbf{k}^{\prime}}=-\sum_{\gamma} \frac{e \mathcal{E}_{\gamma}}{\hbar} \frac{\partial f_{m \mathbf{k}}^{0}}{\partial_{k_{\gamma}}},
$$

where $\Theta_{m \mathbf{k} m^{\prime} \mathbf{k}^{\prime}}$ is the linearized collision integral

$$
\begin{aligned}
\Theta_{m \mathbf{k} m^{\prime} \mathbf{k}^{\prime}}= & \frac{2 \pi}{\mathcal{N} \hbar} \sum_{m^{\prime} \mathbf{q} \mathbf{q}}\left|g_{m \mathbf{k}, m^{\prime} \mathbf{k}+\mathbf{q}}\right|^{2}\left[\delta\left(\epsilon_{m \mathbf{k}}-\hbar \omega_{\nu \mathbf{q}}-\epsilon_{m^{\prime} \mathbf{k}+\mathbf{q}}\right) H_{\mathrm{em}}\right. \\
& \left.+\delta\left(\epsilon_{m \mathbf{k}}+\hbar \omega_{\nu \mathbf{q}}-\epsilon_{m^{\prime} \mathbf{k}+\mathbf{q}}\right) H_{\mathrm{abs}}\right] .
\end{aligned}
$$

Here, $g_{m \mathbf{k}, m^{\prime} \mathbf{k}^{\prime}}$ is the matrix element coupling electron state $m \mathbf{k}$ to another electron state $m^{\prime} \mathbf{k}^{\prime}=m^{\prime} \mathbf{k}+\mathbf{q}$ via emission or absorption of a phonon with wave vector $\mathbf{q}$, polarization $\nu$, and occupancy $N_{v \mathbf{q}}$ given by the Bose distribution. $\mathcal{N}$ is the total number of q-points. The linearized emission and absorption weights are $H_{\mathrm{ems}}=N_{\mathbf{q}}+1-f_{m \mathbf{k}+\mathbf{q}}^{0}$ and $H_{\mathrm{abs}}=$ $N_{\mathbf{q}}+f_{m \mathbf{k}+\mathbf{q}}^{0}$, respectively, if $m^{\prime} \mathbf{k}^{\prime}=m \mathbf{k}$, and $H_{\mathrm{ems}}=-\left(N_{\mathbf{q}}+\right.$ $\left.f_{m \mathbf{k}}^{0}\right)$ and $H_{\text {abs }}=-\left(N_{\mathbf{q}}+1-f_{m \mathbf{k}}^{0}\right)$ if $m^{\prime} \mathbf{k}^{\prime} \neq m \mathbf{k}$. Note that in Eq. (2) we have moved the collision integral to the left-hand side and defined Eq. (3) without the usual minus sign to simplify the following expressions.

In the present study, we restrict the electric field to values where $\Delta f_{m \mathbf{k}} \ll f_{m \mathbf{k}}^{0}$ so that the linearization above is valid. However, in the typical $a b$ initio treatment of transport, the electric field is further assumed to be small enough such that $\partial f_{m \mathbf{k}} / \partial_{k_{\gamma}} \approx \partial f_{m \mathbf{k}}^{0} / \partial_{k_{\gamma}}$, allowing $\Delta f_{m \mathbf{k}}$ to be obtained by an iterative method with only knowledge of $\Theta_{m \mathbf{k} m^{\prime} \mathbf{k}^{\prime}}$ and the equilibrium distribution $f_{m \mathbf{k}}^{0}$ [8]. In the present problem, the field is sufficiently large such that $\partial \Delta f_{m \mathbf{k}} / \partial_{k_{\gamma}} \sim \partial f_{m \mathbf{k}}^{0} / \partial_{k_{\gamma}}$ and the neglected derivative term, $\partial \Delta f_{m \mathbf{k}} / \partial_{k_{y}}$, must be included. This approximation was originally denoted as the 'warm electron' approximation since the excess energy of the electrons over the thermal value can be nonzero while remaining small on that scale [6].

To treat the drift term numerically, we employ a finite difference approximation:

$$
\sum_{\gamma} \frac{e \mathcal{E}_{\gamma}}{\hbar} \frac{\partial \Delta f_{m \mathbf{k}}^{s}}{\partial_{k_{\gamma}}} \approx \sum_{\gamma} \frac{e \mathcal{E}_{\gamma}}{\hbar} \sum_{m^{\prime} \mathbf{k}^{\prime}} D_{m \mathbf{k} m^{\prime} \mathbf{k}^{\prime}, \gamma} \Delta f_{m^{\prime} \mathbf{k}^{\prime}}
$$

where the momentum-space derivative is approximated using the finite-difference scheme given in Ref. [60] and Eq. (8) of Ref. [59].

With these definitions, the steady Boltzmann equation becomes

$$
\begin{aligned}
\sum_{m \mathbf{k}^{\prime}} \Lambda_{m \mathbf{k} m^{\prime} \mathbf{k}^{\prime}} \Delta f_{m^{\prime} \mathbf{k}^{\prime}} & \equiv \sum_{\gamma} \sum_{m \mathbf{k}^{\prime}}\left[\frac{e \mathcal{E}_{\gamma}}{\hbar} D_{m \mathbf{k} m^{\prime} \mathbf{k}^{\prime}, \gamma}+\Theta_{m \mathbf{k} m^{\prime} \mathbf{k}^{\prime}}\right] \Delta f_{m^{\prime} \mathbf{k}^{\prime}} \\
& =\sum_{\gamma} \frac{e \mathcal{E}_{\gamma}}{k_{B} T} v_{m \mathbf{k}, \gamma} f_{m \mathbf{k}}^{0} .
\end{aligned}
$$

Here, we have analytically expanded the gradient of the equilibrium Boltzmann distribution on the right-hand side as $\partial f_{m \mathbf{k}}^{0} / \partial_{k_{\gamma}}=-\left(\hbar v_{m \mathbf{k}, \gamma} / k_{B} T\right) f_{m \mathbf{k}}^{0}$, where $v_{m, \mathbf{k}, \gamma}$ is the group 
velocity along axis $\gamma$ and $k_{B} T$ is the thermal energy. $\Lambda_{m \mathbf{k} m^{\prime} \mathbf{k}^{\prime}}$ is defined as the relaxation operator that combines the drift and scattering operators. Equation (5) shows that the steady Boltzmann equation is now a system of linear equations. The solution, $\Delta f_{m \mathbf{k}}$, can be written symbolically using the inverse relaxation operator:

$$
\Delta f_{m \mathbf{k}}=\sum_{m^{\prime} \mathbf{k}^{\prime}} \Lambda_{m \mathbf{k} m^{\prime} \mathbf{k}^{\prime}}^{-1} \sum_{\gamma}\left(\frac{e \mathcal{E}_{\gamma}}{k_{B} T}\right) v_{m^{\prime} \mathbf{k}^{\prime}, \gamma} f_{m^{\prime} \mathbf{k}^{\prime}}^{0}
$$

Transport properties such as the electrical conductivity can be defined using the steady distribution. In particular, the linear DC conductivity $\sigma_{\alpha \beta}^{\text {lin }}$ can be expressed as

$$
\sigma_{\alpha \beta}^{\text {lin }}=\frac{2 e^{2}}{k_{B} T \mathcal{V}_{0}} \sum_{m \mathbf{k}} v_{m \mathbf{k}, \alpha} \sum_{m^{\prime} \mathbf{k}^{\prime}}\left(\Theta_{m \mathbf{k} m^{\prime} \mathbf{k}^{\prime}}^{-1} v_{m^{\prime} \mathbf{k}^{\prime}, \beta} f_{m^{\prime} \mathbf{k}^{\prime}}^{0}\right),
$$

where the factor of 2 accounts for spin degeneracy and $\mathcal{V}_{0}$ is the supercell volume. The field is applied along the $\beta$ axis and the resulting current is measured along the $\alpha$ axis. The conductivity of Eq. (7) is typically calculated in the cold electron approximation for which $\partial \Delta f_{m \mathbf{k}} / \partial_{k_{\gamma}} \ll \partial f_{m \mathbf{k}}^{0} / \partial_{k_{\gamma}}$ and is thus independent of the electric field.

For sufficiently large fields that $\partial \Delta f_{m \mathbf{k}} / \partial_{k_{\gamma}} \sim \partial f_{m \mathbf{k}}^{0} / \partial_{k_{\gamma}}$, the DC conductivity depends on the electric field and is defined with the relaxation operator:

$$
\sigma_{\alpha \beta}(\mathcal{E})=\frac{2 e^{2}}{k_{B} T \mathcal{V}_{0}} \sum_{m \mathbf{k}} v_{m \mathbf{k}, \alpha} \sum_{m^{\prime} \mathbf{k}^{\prime}} \Lambda_{m \mathbf{k} m^{\prime} \mathbf{k}^{\prime}}^{-1} v_{m^{\prime} \mathbf{k}^{\prime}, \beta} f_{m^{\prime} \mathbf{k}^{\prime}}^{0}
$$

Another important transport quantity, the AC small-signal conductivity $\sigma_{\alpha \beta}^{\omega}$, describes the linear response of the system about a nonequilibrium steady state [22]. With the steady distribution $f_{m \mathbf{k}}^{s}$ being set by a DC field $\mathcal{E}$ as described above, an AC field perturbation along crystal axis $\gamma, \delta \mathcal{E}_{\gamma}(t)=\delta \mathcal{E}_{\gamma} e^{i \omega t}$, induces a fluctuation of the steady distribution $\delta f_{m \mathbf{k}}(t)=$ $\delta f_{m \mathbf{k}}(\omega) e^{i \omega t}$. This fluctuation is governed by the Fourier transformed Boltzmann equation:

$$
\sum_{m^{\prime} \mathbf{k}^{\prime}}(i \omega \mathbb{I}+\Lambda)_{m \mathbf{k} m^{\prime} \mathbf{k}^{\prime}} \delta f_{m^{\prime} \mathbf{k}^{\prime}}=-\sum_{\gamma} \frac{e \delta \mathcal{E}_{\gamma}}{\hbar} \frac{\partial f_{m \mathbf{k}}^{s}}{\partial_{k_{\gamma}}} .
$$

Here, $\mathbb{I}$ is the identity matrix. The fluctuation in the distribution function induces a current fluctuation about the DC value, given as

$$
\delta j_{\alpha}=\frac{2 e}{\mathcal{V}_{0}} \sum_{m \mathbf{k}} v_{m \mathbf{k}, \alpha} \delta f_{m \mathbf{k}} .
$$

The small-signal AC conductivity is defined as the linear coefficient relating the current density variation to the perturbation, $\sigma_{\alpha \beta}^{\omega} \equiv \delta j_{\alpha} / \delta \mathcal{E}_{\beta}$. An explicit expression for $\mathrm{AC}$ conductivity can be obtained by combining the above expressions:

$$
\begin{aligned}
\sigma_{\alpha \beta}^{\omega}= & \frac{2 e}{\mathcal{V}_{0} \delta \mathcal{E}_{\beta}} \sum_{m \mathbf{k}} v_{m \mathbf{k}, \alpha} \sum_{m^{\prime} \mathbf{k}^{\prime}}(i \omega \mathbb{I}+\Lambda)_{m \mathbf{k} m^{\prime} \mathbf{k}^{\prime}}^{-1} \\
& \times\left[\sum_{\gamma}-\frac{e \delta \mathcal{E}_{\gamma}}{\hbar} \frac{\partial f_{m^{\prime} \mathbf{k}^{\prime}}^{s}}{\partial_{k_{\gamma}^{\prime}}}\right] .
\end{aligned}
$$

At equilibrium the steady distribution reduces to the equilibrium distribution $f_{m \mathbf{k}}^{s}=f_{m \mathbf{k}}^{0}$, the kinetic operator reduces to the scattering operator, $\Lambda_{m \mathbf{k} m^{\prime} \mathbf{k}^{\prime}}=\Theta_{m \mathbf{k} m^{\prime} \mathbf{k}^{\prime}}$. By examining Eqs. (7) and (11), we see that at equilibrium the zerofrequency differential conductivity is equal to the linear DC conductivity $\sigma_{\alpha \beta}^{\omega=0}(\mathcal{E}=0)=\sigma_{\alpha \beta}^{\operatorname{lin}}$.

\section{B. Fluctuations about a nonequilibrium steady state}

We now consider fluctuations about a nonequilibrium steady state induced by the stochastic nature of charge carrier scattering. Suppose that the steady state distribution $f_{m \mathbf{k}}^{s}$ is known. Just as in equilibrium, fluctuations in the instantaneous occupation of the quantum states occur. Microscopically, these fluctuations arise because of the stochastic nature of the scattering described by $\Theta_{m \mathbf{k} m^{\prime} \mathbf{k}^{\prime}}$. At steady state, detailed balance requires that the mean flux of particles into every quantum state is zero. However, the flux of particles into or out of a quantum state is a Poissonian process and is characterized by a variance. Therefore, the instantaneous net flux into a quantum state is in general nonzero due to instantaneous imbalance between the incoming and outgoing fluxes [61]. Consequently, the occupancy of quantum states fluctuates under both equilibrium and nonequilibrium conditions.

In the macroscopic limit at which fluctuations are observed in the laboratory, these distribution function fluctuations appear as instantaneous current fluctuations, or equivalently, as electronic noise. A nonrandom characteristic of these fluctuations is the spectral density of current density fluctuations, which, by the Wiener-Khintchine theorem, is related to the single-sided Fourier transform of the autocorrelation of the current density fluctuations [22]:

$$
S_{j_{\alpha} j_{\beta}}(\omega) \equiv\left(\delta j_{\alpha} \delta j_{\beta}\right)_{\omega}=2 \int_{-\infty}^{\infty} \overline{\delta j_{\alpha}(t) \delta j_{\beta}} e^{-i \omega t} d t
$$

where the overbar indicates ensemble average.

We seek to link the macroscopic current density fluctuations to microscopic distribution function fluctuations. Following Ref. [61], we now consider random fluctuations about the nonequilibrium steady state, $\delta f_{m \mathbf{k}}(t)=f_{m \mathbf{k}}(t)-$ $f_{m \mathbf{k}}^{s}$. In contrast to the fluctuations associated with the small signal conductivity, these fluctuations are induced by the stochastic nature of scattering rather than an external perturbation. The corresponding current density fluctuations can be expressed in terms of the fluctuation in the distribution function as in Eq. (10).

It follows that the ensemble average of the correlation function of instantaneous current fluctuations along axes $\alpha$ and $\beta, \overline{\delta j_{\alpha}(t) \delta j_{\beta}}$, can be expressed in terms of the correlation function of the occupancy fluctuations, $\overline{\delta f_{m \mathbf{k}}(t) \delta f_{m_{1} \mathbf{k}_{1}}}$ :

$$
\overline{\delta j_{\alpha}(t) \delta j_{\beta}}=\left(\frac{2 e}{\mathcal{V}_{0}}\right)^{2} \sum_{m \mathbf{k}} \sum_{m_{1} \mathbf{k}_{1}} v_{m \mathbf{k}, \alpha} v_{m_{1} \mathbf{k}_{1}, \beta} \overline{\delta f_{m \mathbf{k}}(t) \delta f_{m_{1} \mathbf{k}_{1}}}
$$

Equation (13) shows that computing the spectral density of current density fluctuations requires calculating the correlations of single particle occupancy fluctuations $\frac{\delta f_{m \mathbf{k}}(t) \delta f_{m_{1} \mathbf{k}_{1}}}{\text {. }}$. This function is known as the time-displaced, two particle correlation function [61]. Through a quantum statistical mechanical treatment, Gantsevich and coauthors have demonstrated that the time-displaced, two particle correlation 
function obeys the same Boltzmann equation as the fluctuation itself [34]:

$$
\frac{\partial}{\partial t} \overline{\delta f_{m \mathbf{k}}(t) \delta f_{m_{1} \mathbf{k}_{1}}}+\sum_{m^{\prime} \mathbf{k}^{\prime}} \Lambda_{m \mathbf{k} m^{\prime} \mathbf{k}^{\prime}} \overline{\delta f_{m^{\prime} \mathbf{k}^{\prime}}(t) \delta f_{m_{1} \mathbf{k}_{1}}}=0 .
$$

The result of Eq. (14) can also be justified less mathematically rigorously but with more physical intuition from Onsager's regression hypothesis (in particular, see Sec. 1 of Ref. [61]).

Solving Eq. (14) requires specifying an initial condition, $\overline{\delta f_{m^{\prime} \mathbf{k}^{\prime}} \delta f_{m_{1} \mathbf{k}_{1}}}$, which is known as the one-time, two-particle correlation function. For a nondegenerate system with a fixed number of particles $N$, Fowler [62] and Lax [33] derived the required condition as

$$
\overline{\delta f_{m \mathbf{k}} \delta f_{m_{1} \mathbf{k}_{1}}}=f_{m \mathbf{k}} \delta_{\mathbf{k k}_{1}} \delta_{m m_{1}}-\frac{f_{m \mathbf{k}} f_{m_{1} \mathbf{k}_{1}}}{N},
$$

where the second term on the right-hand side indicates that a correlation exists between occupancies due to the fixed particle number. With this initial condition for the correlation,
Eqs. (12)-(14) can be combined to express the spectral density of current fluctuations explicitly in terms of solutions to the Boltzmann equation. For a single band, we drop the band index to get

$$
\left(\delta j_{\alpha} \delta j_{\beta}\right)_{\omega}=\left(\frac{2 e}{\mathcal{V}_{0}}\right)^{2} \sum_{\mathbf{k}, \mathbf{k}_{1}} v_{\mathbf{k}, \alpha} v_{\mathbf{k}_{1}, \beta}\left(\delta f_{\mathbf{k}} \delta f_{\mathbf{k}_{1}}\right)_{\omega} .
$$

As with the current density, the spectral density of distribution function fluctuations is related to its analagous correlation function by Fourier transform:

$$
\left(\delta f_{\mathbf{k}} \delta f_{\mathbf{k}_{1}}\right)_{\omega}=\int_{-\infty}^{\infty} \overline{\delta f_{\mathbf{k}}(t) \delta f_{\mathbf{k}_{1}}} \mathrm{e}^{-i \omega t} d t
$$

By exploiting the stationary property of the autocorrelation function, the spectral density can be expressed as [61]

$$
\left(\delta f_{\mathbf{k}} \delta f_{\mathbf{k}_{1}}\right)_{\omega}=2 \Re\left[\sum_{\mathbf{k}^{\prime}}(i \omega \mathbb{I}+\Lambda)_{\mathbf{k} \mathbf{k}^{\prime}}^{-1} \overline{\delta f_{\mathbf{k}^{\prime}} \delta f_{\mathbf{k}_{1}}}\right] .
$$

Combining Eqs. (15), (16), and (18), we obtain the following expression:

$$
\begin{aligned}
S_{j_{\alpha} j_{\beta}}(\omega) & =2\left(\frac{2 e}{\mathcal{V}_{0}}\right)^{2} \mathfrak{R}\left[\sum_{\mathbf{k}} v_{\mathbf{k}, \alpha} \sum_{\mathbf{k}^{\prime}}(i \omega \mathbb{I}+\Lambda)_{\mathbf{k k}^{\prime}}^{-1} \sum_{\mathbf{k}_{1}} v_{\mathbf{k}_{1}, \beta}\left(f_{\mathbf{k}^{\prime}}^{s} \delta_{\mathbf{k}^{\prime} \mathbf{k}_{1}}-\frac{f_{\mathbf{k}^{\prime}}^{s} f_{\mathbf{k}_{1}}^{s}}{N}\right)\right] \\
& =2\left(\frac{2 e}{\mathcal{V}_{0}}\right)^{2} \mathfrak{R}\left[\sum_{\mathbf{k}} v_{\mathbf{k}, \alpha} \sum_{\mathbf{k}^{\prime}}(i \omega \mathbb{I}+\Lambda)_{\mathbf{k} \mathbf{k}^{\prime}}^{-1}\left(f_{\mathbf{k}^{\prime}}^{s}\left(v_{\mathbf{k}^{\prime}, \beta}-V_{\beta}\right)\right)\right]
\end{aligned}
$$

Here, $V_{\beta}$ is the drift velocity along the $\beta$ axis defined as

$$
V_{\beta}=\frac{1}{N} \sum_{\mathbf{k}} v_{\mathbf{k}, \beta} f_{\mathbf{k}}^{s}
$$

where $N=\sum_{\mathbf{k}} f_{\mathbf{k}}$ is the total particle number.

From Eq. (19), it follows that calculating the spectral density of the current fluctuations requires solving the inhomogeneous Boltzmann equation twice. First, the steady occupation function must be obtained using Eq. (6). Then, the Boltzmann equation is solved again with inhomogeneous term $f_{\mathbf{k}}^{s}\left(v_{\mathbf{k}, \beta}-V_{\beta}\right)$ with $f_{m \mathbf{k}}^{s} \equiv f_{m \mathbf{k}}^{0}+\Delta f_{m \mathbf{k}}$. The appropriate Brillouin zone integrations are then performed to calculate the power spectral density.

As a check of the above derivation, consider an equilibrium system for which $\mathcal{E}_{\gamma}=0$ and $V_{\gamma}=0$. The equation is simplified as $f_{\mathbf{k}}^{s}=f_{\mathbf{k}}^{0}$ and $\Lambda_{\mathbf{k k}^{\prime}}=\Theta_{\mathbf{k k}^{\prime}}$. Then, we have

$S_{j_{\alpha} j_{\beta}}(\mathcal{E}=0)=2\left(\frac{2 e}{\mathcal{V}_{0}}\right)^{2} \Re\left[\sum_{\mathbf{k}} v_{\mathbf{k}, \alpha} \sum_{\mathbf{k}^{\prime}}(i \omega \mathbb{I}+\Theta)_{\mathbf{k k}^{\prime}}^{-1} f_{\mathbf{k}^{\prime}}^{0} v_{\mathbf{k}^{\prime}, \beta}\right]$.

With the same simplifications, the equilibrium AC conductivity from Eq. (11) is

$$
\sigma_{\alpha \beta}^{\omega}(\mathcal{E}=0)=\frac{2 e^{2}}{\hbar \mathcal{V}_{0}} \sum_{\mathbf{k}} v_{\mathbf{k}, \alpha}(i \omega \mathbb{I}+\Theta)_{\mathbf{k k}^{\prime}}^{-1}\left[-\frac{\partial f_{\mathbf{k}^{\prime}}^{0}}{\partial_{k_{\beta}^{\prime}}}\right] .
$$

Combining the above expressions, we obtain the familiar Nyquist relationship [27]:

$$
S_{j_{\alpha} j_{\beta}}(\mathcal{E}=0)=4 \frac{k_{B} T_{0}}{\mathcal{V}_{0}} \mathfrak{R}\left[\sigma_{\alpha \beta}^{\omega}(\mathcal{E}=0)\right]
$$

This relationship is formally valid only in equilibrium but remains approximately true in the "cold" electron regime for which $\partial f_{m \mathbf{k}}^{s} / \partial_{\mathbf{k}} \approx \partial f_{m \mathbf{k}}^{0} / \partial_{\mathbf{k}}$ and $\Delta f_{m \mathbf{k}} \ll f_{m \mathbf{k}}^{0}$ such that $\Lambda_{\mathbf{k} \mathbf{k}^{\prime}} \approx$ $\Theta_{\mathbf{k k}^{\prime}}$ and $f_{m \mathbf{k}}^{s} \approx f_{m \mathbf{k}}^{0}$.

\section{NUMERICAL METHODS}

We now describe how to compute the spectral noise power and other quantities using the theory from the previous section. The inputs to the Boltzmann equation are the electronic structure and electron-phonon matrix elements $g_{m \mathbf{k}, m^{\prime} \mathbf{k}+\mathbf{q}}$ calculated using electronic structure packages. First, the electronic structure and electron-phonon matrix elements for GaAs are computed on a coarse $8 \times 8 \times 8$ grid using density functional theory (DFT) and density functional perturbation theory (DFPT), respectively, with QUANTUM ESPRESSO (QE) $[63,64]$. These quantities are then interpolated to finer grids using Wannier interpolation with PERTURBO [65]. PERTURBO includes corrections for polar materials that are necessary in GaAs [66].

The electronic structure calculations using QE employ the same simulation parameters as in Ref. [14]. Briefly, we use a plane wave cutoff of 72 Ryd and a relaxed lattice parameter of $5.556 \AA$. We set the Fermi level to obtain a carrier 
concentration of $10^{15} \mathrm{~cm}^{-3}$ corresponding to a nondegenerate electron gas. We consider only conduction band electronic states within an energy cutoff of $335 \mathrm{meV}$ above the conduction band minimum (CBM). This energy window is larger than the window used in typical electron transport calculations since the present calculations allow the electric field to heat the electrons, leading to occupation at higher energy states. Further increasing the energy window by $50 \mathrm{meV}$ had negligible effect on the observables of interest like spectral noise power.

In PERTURBO, we use a grid of $200 \times 200 \times 200$ for the Wannier interpolated electronic structures and electronphonon matrix elements. The transition rates of Eq. (3) are calculated at $300 \mathrm{~K}$. We consider convergence by determining the change in the spectral noise power at the maximum electric field for which the $\Delta f_{m \mathbf{k}} \ll f_{m \mathbf{k}}^{0}$ assumption is satisfied. Numerical experimentation shows that this condition is satisfied for $\mathcal{E} \lesssim 800 \mathrm{~V} \mathrm{~cm}^{-1}$. The spectral noise power at $800 \mathrm{~V} \mathrm{~cm}^{-1}$ using the $200 \times 200 \times 200$ grid differs by less than $1 \%$ from the value obtained on a grid with twice the number of grid points. The delta function in the electronphonon matrix elements is approximated with a Gaussian with a $10 \mathrm{meV}$ broadening parameter [14]. Decreasing the broadening to $6 \mathrm{meV}$ changed the spectral noise power at 800 $\mathrm{V} \mathrm{cm}^{-1}$ by less than $3 \%$.

While PERTURBO performs the Wannier interpolation for the electron-phonon interaction on fine grids [59], it does not explicitly construct the collision matrix of Eq. (3). Instead, the mobility is computed using an iterative scheme under the cold electron approximation [8]. We found that this iterative method was numerically unstable for the warm electron case. We instead solved the linear system using the generalized minimal residual (GMRES) algorithm as implemented in the scientific PYTHON library [67]. The matrix was constructed by modifying PERTURBO to output the elements of Eq. (3).

As described in Sec. II, the derivative term corresponding to particle drift in an electric field $\mathcal{E}$ is approximated by a finite difference matrix. Boundary conditions must be applied to points that do not have a full set of first-nearest neighbors. To do so, we assume that these points have zero occupation by removing the contributions of these states to the finite difference matrix. The energy window is selected so that these boundary states indeed have negligible population, also ensuring that scattering induced by the collision matrix $\Theta_{m \mathbf{k} m^{\prime} \mathbf{k}^{\prime}}$ for these states can be neglected.

With the collision and drift matrices computed, we then construct the relaxation operator $\Lambda_{m \mathbf{k} m^{\prime} \mathbf{k}^{\prime}}$, Eq. (5). The steadystate distribution is obtained by solving the resulting linear system given by Eq. (6). We then solve Eq. (19) with the inhomogenous term constructed from the previously computed steady-state distribution as input. For this second Boltzmann equation, we include an $i \omega$ term on the diagonal of the linear system which corresponds to the Fourier transformed time derivative. Finally, the spectral density is computed as a Brillouin zone integration over the distribution that solves the second Boltzmann equation. The calculation of the AC mobility proceeds in a similar way as for the steady distribution except with the addition of the $i \omega$ term.

In this work, we apply our method to GaAs. Owing to computational limitations, we account for first-order electron- phonon (1ph) processes and neglect higher-order interactions that are reported to play a role in GaAs [16]. Also, recently studies report the effect of quadrupole electron-phonon interactions on electron transport $[68,69]$. In particular, the work of Ref. [69] predicted a significant correction to the mobility in GaAs limited by acoustic mode scattering. Our calculations were performed at $300 \mathrm{~K}$ at which the scattering is dominated by polar optical phonons, and so we neglected quadrupole interactions. Finally, we note that the method described above is easily extendable to other technologically interesting semiconductors. In particular, first-principles calculations of electron-phonon interactions in $\mathrm{Si}$, InP, and $\mathrm{Ge}$ are now routine, noise calculations in these materials are the subject of ongoing work.

\section{RESULTS}

\section{A. Transport}

We begin by examining the steady state distribution and associated transport observables in the cold and warm electron regimes. Figure 1(a) plots the deviational steady state distribution functions under the two approximations versus wave vector parallel to the electric field, $k_{x}$. We refer to this direction as the longitudinal direction. At low fields $\mathcal{E}<100 \mathrm{~V} \mathrm{~cm}^{-1}$, the solutions are nearly identical, but as the field increases, differences in the distribution functions emerge. Under the cold electron approximation, Eq. (6) shows that $\Delta f_{m \mathbf{k}}$ is required to possess odd symmetry about the Brillouin zone center because $\partial f_{m \mathbf{k}}^{0} / \partial_{\mathbf{k}}$ is odd with respect to $k_{x}$ while the scattering matrix is even $\left(\Theta_{\mathbf{k k}^{\prime}}=\Theta_{-\mathbf{k}-\mathbf{k}^{\prime}}\right)$; this symmetry is evident in Fig. 1(a). In contrast, in the warm electron case the electrons can be heated and the solution becomes asymmetric with increasing field.

The transport properties of the warm electron distribution differ from those of the cold distribution because warm electrons in the high energy tail are able to emit optical phonons and hence exhibit higher scattering rates. As reported previously [14], the predicted mobility of GaAs exceeds the experimental mobility owing to the exclusion of higher-order phonon scattering processes and the lower calculated effective mass $\left(0.055 m_{0}\right)$ compared to experiment $\left(0.067 m_{0}\right)$ [16].

Therefore, to facilitate comparison we examine the DC mobility normalized by its low-field value in Fig. 1(b). The low-field value of the computed mobility is 17420 $\mathrm{cm}^{2} \mathrm{~V}^{-1} \mathrm{~s}^{-1}$. At low fields $\mathcal{E}<100 \mathrm{~V} \mathrm{~cm}^{-1}$, the mobility under the warm and cold electron approximations agrees to within $1 \%$. At $\mathcal{E}=800 \mathrm{~V} \mathrm{~cm}^{-1}$, the DC mobility of the warm electrons has decreased by more than $10 \%$. This behavior is qualitatively consistent with the sublinear current voltage characteristic (CVC) of $n$-type GaAs [22], or a decrease in mobility with increasing electron temperature. The field dependence of the normalized mobility shows favorable comparison to experiment, implying that our calculation is properly capturing the heating with the field.

In addition to steady quantities, the small-signal $\mathrm{AC}$ mobility can be computed as in Eq. (11). Figure 1(c) presents the small-signal AC mobility for the warm electron gas versus frequency for several electric fields. At zero frequency, the equilibrium $\mathrm{AC}$ mobility is equal to the equilibrium DC 

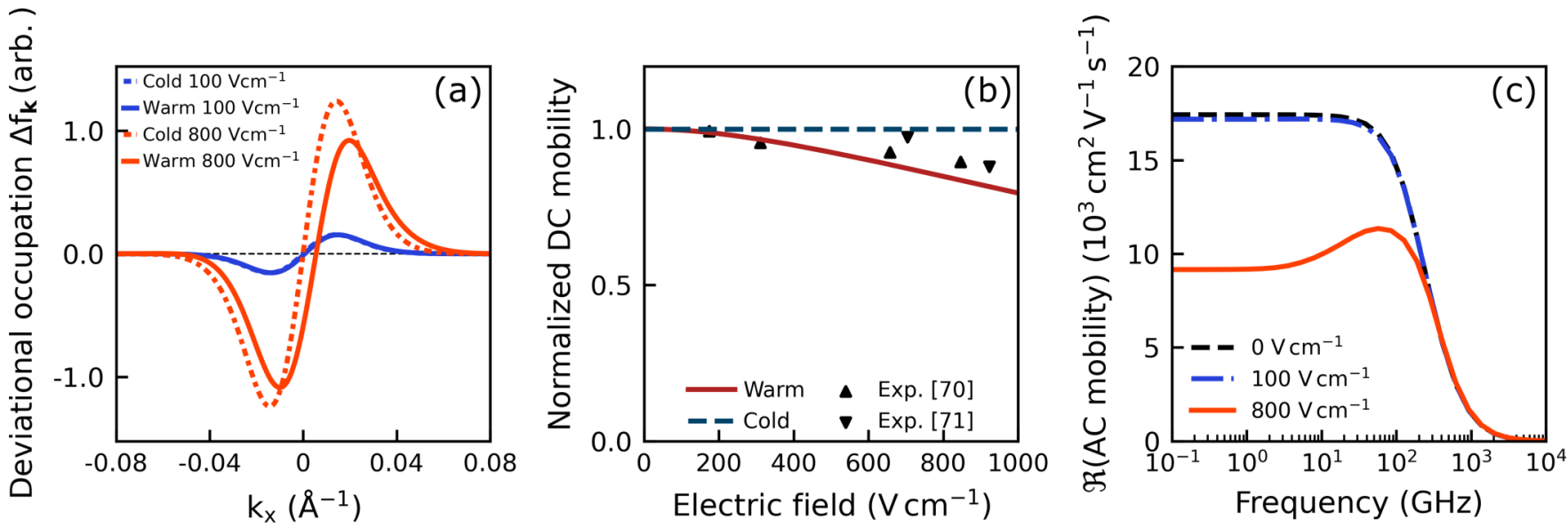

FIG. 1. (a) Deviational occupation $\Delta f_{k}$ in GaAs at $300 \mathrm{~K}$ under the cold (dotted lines) and warm (solid lines) electron approximations versus longitudinal wave vector $k_{x}$. Curves plotted for $\mathcal{E}=100$ (blue) and $800 \mathrm{~V} \mathrm{~cm}^{-1}$ (orange). The dashed black line is a guide to the eye. (b) Normalized longitudinal ( $\|$ ) DC mobility versus electric field of the cold (dashed blue line) and warm electrons (solid red line). The heating of the electrons leads to a decreased mobility. The trend of the normalized mobility agrees well with experiments: Fig. 1, Ref. [70] (Upward black triangles) and Fig. 4, Ref. [71] (Downward black triangles). (c) Real part of the longitudinal small-signal AC mobility versus frequency for equilibrium (dashed black line), $\mathcal{E}=100$ (dash-dot blue line) and $800 \mathrm{~V} \mathrm{~cm}^{-1}$ (solid orange line) under the warm electron approximation. The AC mobility exhibits spectral features at frequencies that are characteristic of the inverse momentum and energy relaxation times (see Sec. IV C).

mobility, as expected. The decrease of the AC mobility with electric field at is also consistent with the trend observed in the DC mobility. At $f \sim 1 \mathrm{THz}$, the AC field frequency exceeds the phonon-mediated scattering rates which redistribute the electrons, and thus the AC mobility rolls off at all fields. This result reflects the electrical response transitioning from a purely resistive to a purely reactive regime as the frequency exceeds the highest scattering rates.

The frequency dependence of the AC mobility indicates the relevant timescales of momentum and energy relaxation [72]. In particular, for $800 \mathrm{~V} \mathrm{~cm}^{-1}$, we observe a lower value of the AC mobility at low frequency, followed by a maximum at around $100 \mathrm{GHz}$. This feature is due to energy exchange with phonons and will be discussed in Sec. IV C.

\section{B. Diffusion noise}

We now calculate the spectral density of current fluctuations from the nonequilibrium steady state in GaAs. Figure 2(a) shows the spectral density of longitudinal current fluctuations versus electric field at an observation frequency of $1 \mathrm{MHz}$, far smaller than any scattering rate. At equilibrium, the noise is given by the Nyquist relation, Eq. (23). It is conventional to report the spectral density normalized
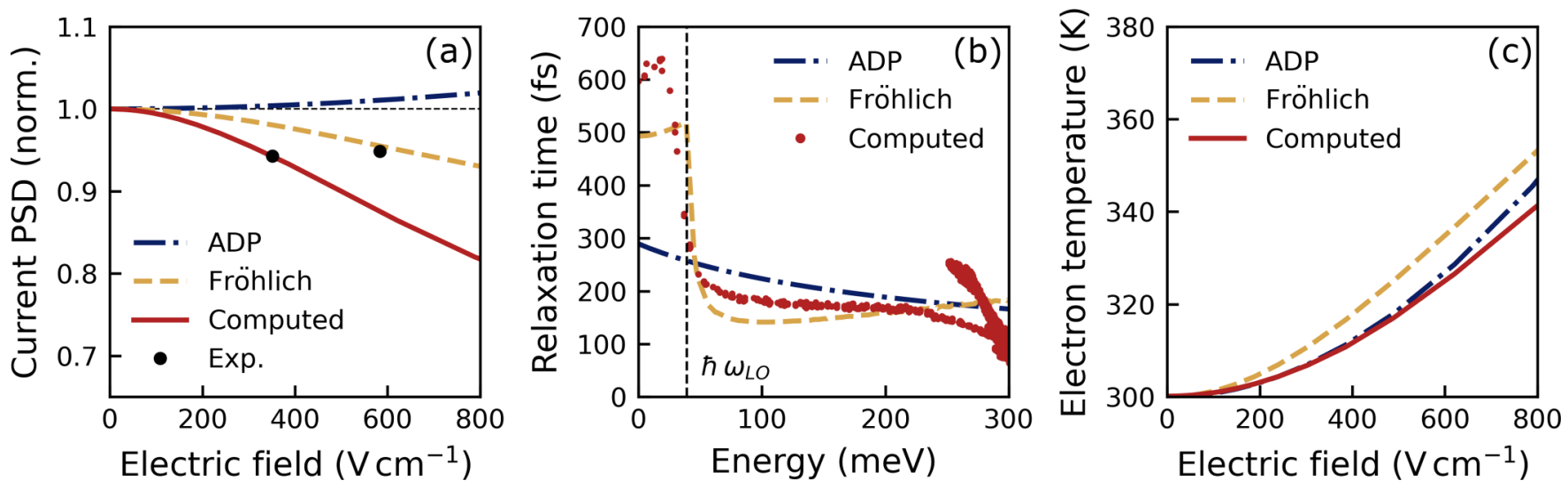

FIG. 2. (a) Spectral density of longitudinal current density fluctuations (solid red line) normalized to the Nyquist value vs electric field along with Davydov spectral densities calculated using ADP (dash-dot blue line) and Fröhlich (dashed yellow line). At equilibrium, the noise agrees with Nyquist-Johnson noise (dotted black line). The ab initio calculation predicts a steeper decrease in current PSD with field compared to the approximations. The symbols correspond to experimental measurements (Fig. 11, Ref. [73]). (b) Relaxation time versus energy above conduction band minimum for GaAs at $300 \mathrm{~K}$ using ADP (dash-dot blue line), Fröhlich potential (dashed yellow line), and computed (red circles). The energy of the zone-center LO phonon is shown for reference (dashed black line). (c) Effective electron gas temperature versus electric field for ADP (dash-dot blue line), Fröhlich (dashed yellow line), and computed (solid red line). The magnitude of electron heating is similar among the various calculations. 

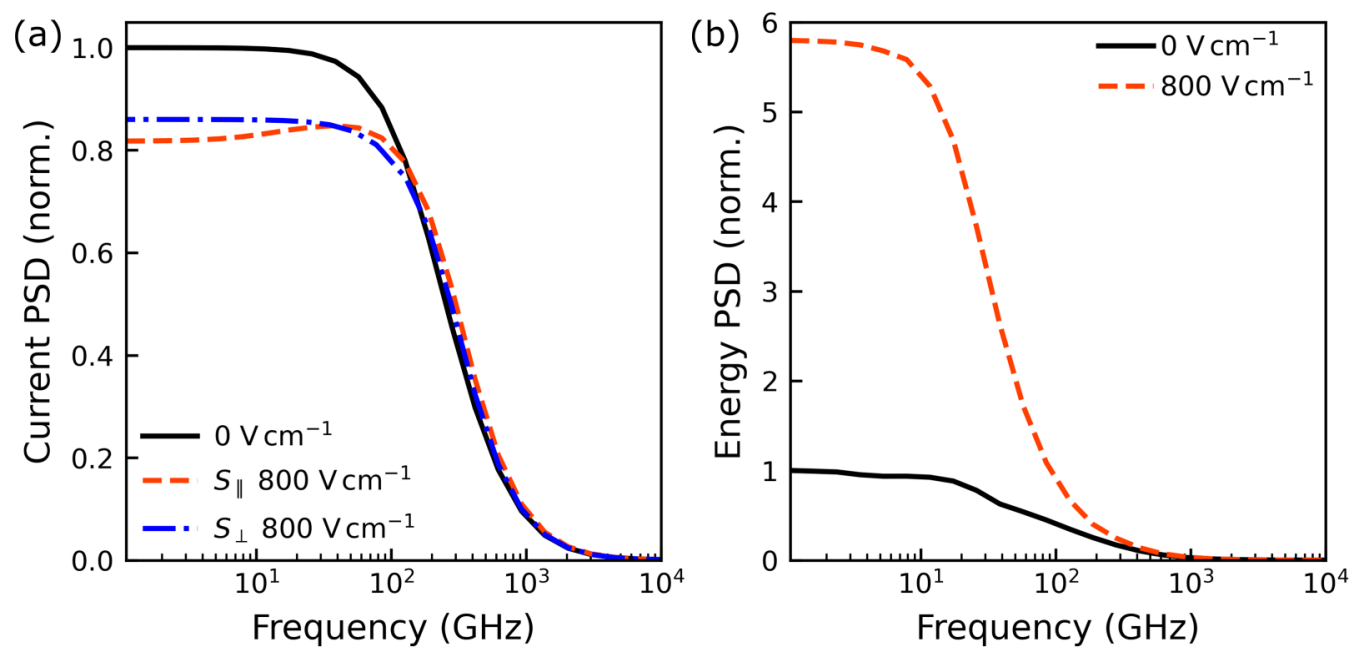

FIG. 3. (a) Computed power spectral density (PSD) of longitudinal ( $\|$, dashed orange line) and transverse ( $\perp$, dashed-dotted blue line) current density fluctuations versus frequency at $\mathcal{E}=800 \mathrm{~V} \mathrm{~cm}^{-1}$, along with the Nyquist-Johnson prediction for $\mathcal{E}=0$ (solid black line). (b) Spectral density of energy fluctuations vs frequency at equilibrium (solid black line), $\mathcal{E}=800 \mathrm{~V} \mathrm{~cm}^{-1}$ (dashed orange line). The time scale for electron temperature fluctuations sets the upper frequency limit for the convective mechanism.

to the Nyqist value to allow comparison between samples of different carrier density [23].

As the electric field increases, the computed noise decreases below the Nyquist value. Few experimental studies of noise in GaAs cover the fields of present interest, but reasonable agreement is observed with measurements by Bareikis et al. [73]. We note that a decrease with field is observed in other studies in GaAs [70,74] though the sparsity of data in the relevant electric field range prevents direct comparison.

To better understand the decreasing trend, we use an approximate solution of the Boltzmann equation for an electron gas interacting quasi-elastically with a thermal phonon bath $[25,75]$. Under the quasi-elastic approximation, the distribution function is expanded in momentum space using Legendre polynomials. Because the distribution is nearly isotropic in momentum space under quasielastic scattering, only the two lowest Legendre polynomials need be retained [76]; the zeroth-order term gives the occupancy versus energy and is known as the Davydov distribution. The model is parametrized by the energy dependence of the momentum and energy relaxation times, $\tau$ and $\tau_{\epsilon}$, respectively, and the inelasticity ratio $\tau / \tau_{\epsilon}$ [22]. Once these parameters are specified, the Davydov distribution can be computed and used with Eq. (19) to calculate the spectral density of current fluctuations [61].

Approximate analytic expressions for the electron relaxation times in semiconductors are available [3]. Previous works have calculated the Davydov distribution for a powerlaw energy dependence of the relaxation times such as that from the acoustic deformation potential (ADP) [77-79]. However, in GaAs at room temperature, the long-ranged Fröhlich interaction with longitudinal optical (LO) phonons is the dominant scattering mechanism $[14,15]$.

In Fig. 2(a), we compare the $a b$ initio longitudinal spectral density to that predicted using the Davydov distribution with the ADP and Fröhlich scattering rates. The approximate relaxation times have been scaled to match the computed low-field mobility, and the inelasticity ratio has been selected using an estimation of the energy and momentum relaxation times (see Fig. 3). The computed spectral density is observed to decrease monotonically with the electric field. This decrease is captured qualitatively by the Fröhlich calculation. In contrast, the ADP noise increases monotonically with field.

These trends can be understood in terms of the differing energy dependencies of the relaxation times in the various approximations. Figure 2(b) shows the phonon-mediated relaxation times versus energy for electrons in GaAs at $300 \mathrm{~K}$ for the three cases. Below the zone-center LO phonon energy $\hbar \omega_{\mathrm{LO}} \sim 35 \mathrm{meV}$, the computed relaxation times are set by LO phonon absorption [14]. Above the LO phonon energy, LO emission becomes dominant and the relaxation times sharply decrease to a value that remains roughly constant until electron energies are near the $L$-valley minimum at $\sim 0.25 \mathrm{eV}$ above the CBM. This absorption-to-emission transition is qualitatively captured by the Fröhlich approximation. The ADP relaxation times agree reasonably well with the computed ones in the emission-dominated region but do not exhibit the absorption-to-emission transition.

The electric field dependence of the spectral noise power reflects the balance between the growth of scattering rates with electron energy and the heating of the electron gas by the DC field [80]. To understand this balance, we examine the effective electron temperature of the steady distribution for the three cases in Fig. 2(c). The effective electron temperature is calculated as the temperature of a Maxwell-Boltzmann distribution that yields the same energy density as the steady nonequilibrium distribution. At low fields $\mathcal{E}<100 \mathrm{~V} \mathrm{~cm}^{-1}$, the temperature is equal to the lattice temperature. As the electric field increases, the effective temperature increases, corresponding to occupancy at higher energies and increased scattering rates. Near equilibrium where the mobilities are equivalent, the temperature rise predicted from each approximation is similar, but at higher fields, the $a b$ initio calculation predicts a slightly lower temperature than do either the ADP or Fröhlich approximations. 
As the electron gas heats, higher energy states are occupied and thus the spectral noise power, Eq. (19), includes contributions from fluctuations in those states; hence, the spectral noise power may increase on heating. On the other hand, at these high energies, the scattering events which damp out fluctuations are more frequent, tending to decrease the noise. The competition between these mechanisms sets the trends shown in Fig. 2(a). For both Fröhlich and the present calculations, the sharp increase in scattering rates associated with the absorption-to-emission transition dominates, and the spectral density decreases monotonically with electric field. In contrast, the ADP approximation shows increasing noise with electric field as the heating of the electrons dominates the weak increase of the scattering rates.

The evolution of the spectral density with electric field demonstrates the sensitivity of the spectral noise power to the energy dependence of the scattering rates. Although the mobility at equilibrium is equivalent for all three cases, the nonequilibrium noise behavior exhibits qualitatively different trends depending on the energy dependence and inelasticity of the scattering mechanisms.

\section{Spectral noise power}

The nonequilibrium noise exhibits spectral features that are not present in the Nyquist-Johnson case. Figure 3(a) shows the spectral density of longitudinal (L) and transverse (T) current fluctuations (relative to the electric field axis) versus frequency at $\mathcal{E}=800 \mathrm{~V} \mathrm{~cm}^{-1}$. There are several notable features of the spectral density in this figure. First, the spectral density is constant at low frequencies and rolls off as frequency increases, decreasing to $50 \%$ of its low-frequency value at $300 \mathrm{GHz}$. Secondly, an anisotropy exists between the longitudinal and transverse spectral densities. Finally, the longitudinal noise exhibits a nonmonotonic trend for frequencies around $50 \mathrm{GHz}$, similar to that observed for the AC mobility in Fig. 1(c). Spectroscopic measurements of the noise power at these frequencies have not been performed, but these trends are qualitatively similar to those observed in recent Monte Carlo simulations [58].

We discuss each of these points in turn. Consider first the noise at equilibrium. The zero-field curve shows that the longitudinal and transverse spectral densities are equal and coincide with the Nyquist-Johnson value, Eq. (23). As with the AC mobility, the spectral density rolls off at frequencies exceeding the phonon-mediated scattering rates because the electronic system cannot redistribute in response to the fluctuation. This roll-off behavior has been noted previously [6] and has also been observed for phonon thermal conductivity (see Fig. 1(b) in Ref. [81]).

Now consider the noise with $\mathcal{E}=800 \mathrm{~V} \mathrm{~cm}^{-1}$. A similar roll-off with increasing frequency as the equilibrium case is observed. At low frequency, both the longitudinal and transverse spectral densities are lower than the Nyquist value because of the increased electron temperature. However, an anisotropy exists in the spectral densities. The origin of this feature is the 'convective' mechanism [22,25,82] and can be understood by decomposing the current fluctuations into two sources. The first is the fluctuation of the drift velocity, induced by stochastic transitions between states of differing group velocity. The second is the fluctuation of the electron temperature, induced by random energy exchange with the thermal phonon bath. Under nonequilibrium conditions, these fluctuations couple. As the gas is heated by the electric field, the fluctuating current induces a variation in the Joule heating. The resulting electron temperature fluctuation changes the conductivity, which in turn modifies the current. This coupling only exists for fluctuations longitudinal to the electric field because transverse fluctuations do not affect Joule heating. In sublinear CVC materials such as GaAs, the conductivity decreases with electron temperature, and the convective mechanism suppresses longitudinal fluctuations. This feature is indeed observed in Fig. 3(a).

The convective mechanism is only present at frequencies $\omega \tau_{\epsilon} \ll 1$, where $\tau_{\epsilon}$ is the energy relaxation time. As discussed above, the local maxima from the convective contribution appears at $\omega \tau_{\epsilon}=1$ in the longitudinal direction (see Ref. [22], Chap. 7). The energy relaxation time can also be extracted by calculating the spectral density of electron temperature fluctuations versus frequency. This calculation is the energy analog of Eq. (19), where the relevant state quantity is the energy instead of the group velocity. Figure 3(b) shows the spectral density of energy fluctuations versus frequency for several electric fields. At low frequencies, $f<10 \mathrm{GHz}$, the spectral density increases with field as the temperature fluctuations rise with higher Joule heating. At higher frequencies, $f \sim 50 \mathrm{GHz}$, the energy fluctuations decrease to $50 \%$ of their low-frequency values and begin to converge for the two fields shown. This convergence signifies that the temperature of the electron gas cannot change sufficiently rapidly due to its finite thermal capacitance. Consequently, the convective noise mechanism is removed and the anisotropy of the densities in Fig. 3(a) also disappears; the longitudinal and transverse spectral densities converge. The convective mechanism is also responsible for the nonmonotonic trend of the $\mathrm{AC}$ mobility seen in Fig. 1(c).

\section{Quasi-elastic scattering}

The present formalism for electronic noise permits the study of the microscopic processes responsible for electronic noise in a manner that is difficult to obtain by other methods. As an example, consider the spectral features present in Fig. 3. Comparing the frequency where the current power spectral density and energy power spectral density reach half of their low-frequency values ( $300 \mathrm{GHz}$ versus $50 \mathrm{GHz}$, respectively), the energy relaxation time is inferred to be around 6 times longer than the momentum relaxation time, implying that the quasielastic assumption is valid. This observation is surprising given the well-known dominance of high-energy LO phonon emission in GaAs [14] and that inelasticity is expected only when the physical temperature is comparable to the Debye temperature [6]. Analytical treatments of noise under dominant LO phonon coupling typically assume strongly inelastic interactions between the electrons and lattice (see Sec. 3.8 of Ref. [25], Sec. 7.3 of Ref. [22], or Ref. [83]).

We identify the origin of this discrepancy by examining how individual scattering events contribute to the momentum and energy relaxation of the electron system to the phonons. These transfers can be expressed as sums over each of the 

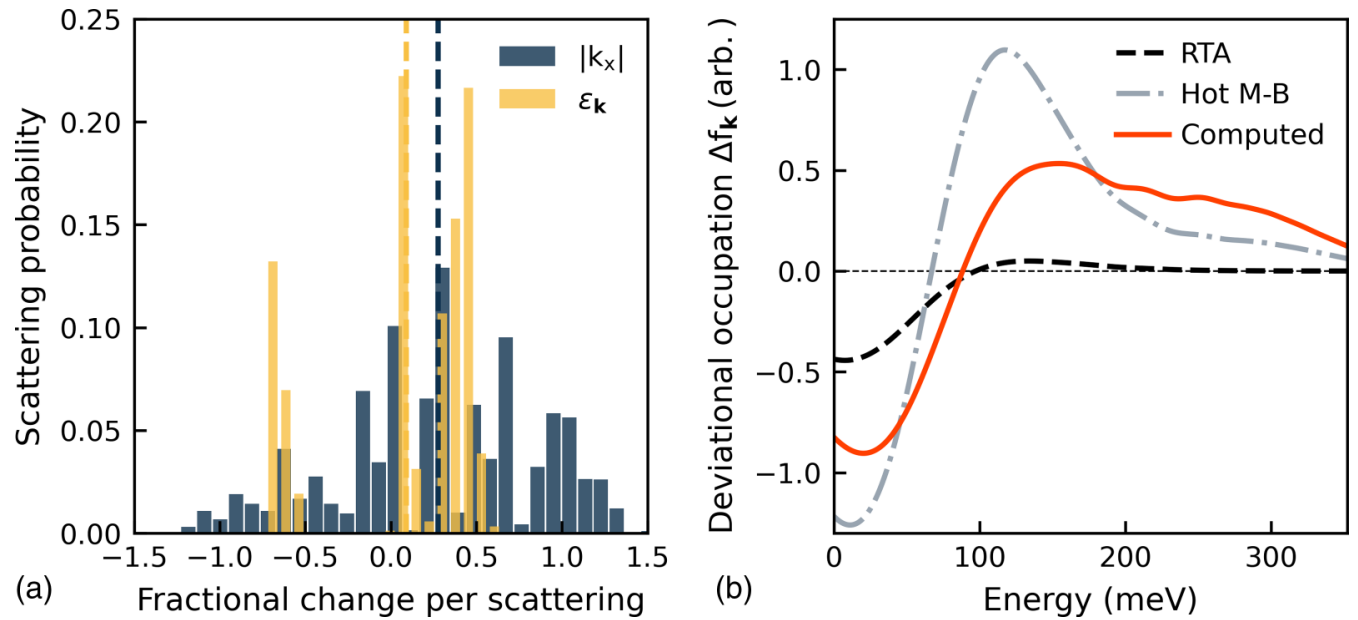

FIG. 4. (a) Probability histograms of longitudinal momentum loss $R_{\mathbf{k}}$ (blue bars) and energy loss $R_{\epsilon_{\mathbf{k}}}$ (yellow bars) normalized by the thermal averages at $800 \mathrm{~V} \mathrm{~cm}^{-1}$. The dashed lines represent the average transfer per scattering event. At this field, the average fractional dissipation of longitudinal momentum is $\sim 3 \times$ larger than that for energy. (b) Deviational occupation $\Delta f_{\mathbf{k}}$ in GaAs at $300 \mathrm{~K}$ versus energy calculated under the RTA (dashed black line), hot Maxwell-Boltzmann (dashed-dotted grey line), and ab initio warm electron approximation (solid orange line) at $800 \mathrm{~V} \mathrm{~cm}^{-1}$. The dashed black line is added as a guide to the eye. Neither the RTA nor the Maxwell-Boltzmann capture the hot electron tail.

electron-phonon scattering processes in the collision integral weighted by the energy and momentum of the mediating phonon. Every electronic state in the BZ is coupled via phonons to other states; by summing over all possible scattering processes, we obtain the average energy and momentum exchanged in a single scattering event. More precisely, the fractional change in momentum and energy per scattering event are calculated from

$$
R_{x}=\frac{1}{\Theta_{\mathbf{k k}}\langle|x|\rangle} \sum_{\mathbf{k}^{\prime}} \Delta x \Theta_{\mathbf{k}^{\prime} \mathbf{k}},
$$

where $x=k_{x}, \epsilon_{k}$ and $\Delta x=x-x^{\prime}$ is the difference in the state quantity between $\mathbf{k}$ and $\mathbf{k}^{\prime} .\langle|x|\rangle$ denotes the thermal average magnitude of the relevant quantity; $\Theta_{\mathbf{k}^{\prime} \mathbf{k}}$ represents the component of the diagonal element of the collision matrix (the scattering rate) corresponding to scattering from $\mathbf{k}$ to $\mathbf{k}^{\prime}$; and other variables carry same meaning as defined in Sec. II.

These fractional changes at $800 \mathrm{~V} \mathrm{~cm}^{-1}$ are plotted as a probability histogram in Fig. 4(a). In this figure, we have binned each state in the BZ by the value of $R_{\mathbf{k}}$ and $R_{\epsilon_{\mathrm{k}}}$. For all the states in a given bin, we calculate the probability of scattering $\mathcal{P} \propto \sum_{\text {bin }} \Theta_{\mathbf{k k}} f_{m \mathbf{k}}^{s}$ (the final quantity is normalized to unity). The horizontal position indicates the average fractional change in energy or momentum induced by the event. Positive values of the fractional change correspond to net transfers to the lattice, or dissipation, while negative values correspond to transfers to the electrons, or accumulation. The height of a bar represents the population-weighted probability of scattering in a given time interval.

Figure 4(a) reveals several important features. First, energy transfers are clustered into two groups. The grouping of accumulation events around -0.75 corresponds to the $\sim 35 \mathrm{meV}$ energy gain associated with LO absorption, which dominates scattering of electrons below the emission threshold $\hbar \omega_{\mathrm{LO}}$. The relatively disperse grouping of the dissipation events reflects a balance between LO emission and absorption for states above the threshold. Second, in contrast to energy transfers, momentum transfers grow with the wave vector of the mediating phonon. Consequently, a broader and more disperse distribution of momentum transfers is available. Finally, the balance between dissipation and accumulation differs between energy and momentum. In equilibrium, these processes are balanced, but at $800 \mathrm{~V} \mathrm{~cm}^{-1}$, the net transfers for both quantities are dissipative as the warm electrons transfer excess momentum and energy to the lattice. The dashed lines in the figure represent the average fractional transfer per scattering event and indicate that the net momentum dissipation exceeds the energy dissipation by around a factor of 3. This imbalance is partly responsible for the disparate time scales of energy and momentum relaxation observed in Fig. 3.

The second contributing factor to the relatively long energy relaxation time is the presence of a hot electron tail in the calculated distribution. In Fig. 4(b), we plot the steady deviation distribution, $\Delta f_{s}$, calculated under the warm electron approximation using the full $e$-ph scattering matrix versus energy. For reference, the corresponding distributions for a hot Maxwell-Boltzmann at the nonequilibrium electron temperature and a 'relaxation-time distribution' obtained under the warm electron approximation with only the on-diagonal elements of the scattering matrix. The ab initio treatment predicts a hot electron tail that is not observed with either approximate method. Although representing only a small fraction of the population, these hot electrons are at energies $5-10 \times$ the thermal average value. Consequently, many scattering events are needed to return these electrons to equilibrium, further increasing the energy relaxation time. The result is that the quasi-elastic approximation is unexpectedly accurate despite the inelastic nature of optical phonon scattering, and thus explaining the features in the spectral noise power and $\mathrm{AC}$ mobility. 


\section{SUMMARY AND FUTURE OUTLOOK}

The primary numerical tools used to study electronic noise are Monte Carlo (MC) methods [56-58,84,85]. These simulators have many advantages, including the ability to incorporate realistic device geometries and space charge effects through coupled Poisson solvers, and they are thus useful to interpret experimental measurements on devices. However, MC studies rely upon semi-empirical models of scattering and electronic structure that require parameters such as deformation potentials, sound velocities, effective masses, and energy gaps to be specified and calibrated against experiment. The methods are thus most useful for well-characterized materials for which these empirical models are available.

A parallel development in the transport field has been the introduction of $a b$ initio methods to study low-field transport phenomena without adjustable parameters. These methods enable not only the computation of low-field transport properties such as electronic mobility [8,9] and phonon thermal conductivity [86] but also an understanding of the microscopic scattering processes that underlie these macroscopic properties and prediction of the properties of new materials. However, thus far these methods have been restricted to the low-field, cold electron regime.

In this work, we have described an ab initio theory of electronic noise for warm electrons in semiconductors. The method requires no adjustable parameters, with the phonon dispersion, band structure, and electron-phonon coupling calculated from first principles. Further, this method permits the study of transport even when the electrons are not in equilibrium with the lattice, being free of the cold electron approximation used in previous transport studies. To demonstrate the method, we performed calculations in GaAs, a technologically relevant material, and demonstrated that the spectral features of the AC mobility and current noise are linked to the disparate time scales of energy and momentum relaxation. The quasi-elastic approximation is unexpectedly accurate in GaAs despite the dominance of polar optical phonon scattering. Our work paves the way for first-principles studies of electronic noise in other semiconductors that will advance the study of transport phenomena and applications of low-noise semiconductor devices.

\section{ACKNOWLEDGMENTS}

The authors thank Jin-Jian Zhou, I-Te Lu, Vatsal Jhalani, and Marco Bernardi for assistance with PERTURBO and useful discussions. This work was supported by AFOSR under Grant No. FA9550-19-1-0321. This material is based upon work supported by the National Science Foundation Graduate Research Fellowship under Grant No. DGE-1745301. Any opinions, findings, and conclusions or recommendations expressed in this material are those of the author and do not necessarily reflect the views of the National Science Foundation.
[1] J. W. Ziman, Electrons and Phonons: The Theory of Transport Phenomena in Solids (Oxford University Press, Oxford, 1960).

[2] D. K. Ferry, Semiconductor Transport (CRC Press, London, 2000).

[3] M. Lundstrom, Fundamentals of Carrier Transport (Cambridge University Press, Cambridge, 2000).

[4] G. Chen, Nanoscale Energy Transport and Conversion: A Parallel Treatment of Electrons, Molecules, Phonons, and Photons, MIT-Pappalardo Series in Mechanical Engineering (Oxford University Press, New York, 2005).

[5] G. D. Mahan, Condensed Matter in a Nutshell (Princeton University Press, Princeton, 2011).

[6] E. Conwell, High Field Transport in Semiconductors (Academic Press, New York, 1967).

[7] B. Liao, J. Zhou, B. Qiu, M. S. Dresselhaus, and G. Chen, $\mathrm{Ab}$ initio study of electron-phonon interaction in phosphorene, Phys. Rev. B 91, 235419 (2015).

[8] M. Bernardi, First-principles dynamics of electrons and phonons, Eur. Phys. J. B 89, 239 (2016).

[9] F. Giustino, Electron-phonon interactions from first principles, Rev. Mod. Phys. 89, 015003 (2017).

[10] M. Bernardi, D. Vigil-Fowler, Johannes Lischner, J. B. Neaton, and S. G. Louie, Ab Initio Study of Hot Carriers in the First Picosecond after Sunlight Absorption in Silicon, Phys. Rev. Lett. 112, 257402 (2014).

[11] S. Poncé, E. R. Margine, and F. Giustino, Towards predictive many-body calculations of phonon-limited carrier mobilities in semiconductors, Phys. Rev. B 97, 121201(R) (2018).
[12] M. Fiorentini and N. Bonini, Thermoelectric coefficients of $n$-doped silicon from first principles via the solution of the boltzmann transport equation, Phys. Rev. B 94, 085204 (2016).

[13] W. Li, Electrical transport limited by electron-phonon coupling from boltzmann transport equation: An ab initio study of si, al, and $\operatorname{mos}_{2}$, Phys. Rev. B 92, 075405 (2015).

[14] Jin-Jian Zhou and Marco Bernardi, Ab initio electron mobility and polar phonon scattering in gaas, Phys. Rev. B 94, 201201 (2016).

[15] T.-H. Liu, J. Zhou, B. Liao, D. J. Singh, and G. Chen, First-principles mode-by-mode analysis for electron-phonon scattering channels and mean free path spectra in gaas, Phys. Rev. B 95, 075206 (2017).

[16] N.-E. Lee, J.-J. Zhou, H.-Y. Chen, and M. Bernardi, Ab initio electron-two-phonon scattering in GaAs from next-toleading order perturbation theory, Nat. Commun. 11, 1607 (2020).

[17] N.-E. Lee, J.-J. Zhou, L. A. Agapito, and M. Bernardi, Charge transport in organic molecular semiconductors from first principles: The bandlike hole mobility in a naphthalene crystal, Phys. Rev. B 97, 115203 (2018).

[18] V. A. Jhalani, J.-J. Zhou, and M. Bernardi, Ultrafast hot carrier dynamics in gan and its impact on the efficiency droop, Nano Lett. 17, 5012 (2017).

[19] J. Ma, A. S. Nissimagoudar, and W. Li, First-principles study of electron and hole mobilities of si and gaas, Phys. Rev. B 97, 045201 (2018). 
[20] J. Sun, H. Shi, T. Siegrist, and David J. Singh, Electronic, transport, and optical properties of bulk and mono-layer pdse2, Appl. Phys. Lett. 107, 153902 (2015).

[21] T.-H. Liu, B. Song, L. Meroueh, Z. Ding, Q. Song, J. Zhou, M. Li, and G. Chen, Simultaneously high electron and hole mobilities in cubic boron- $\mathrm{V}$ compounds: BP, BAs, and BSb, Phys. Rev. B 98, 081203(R) (2018).

[22] H. L. Hartnagel, R. Katilius, and A. Matulionis, Microwave Noise in Semiconductor Devices (Wiley, New York, 2001).

[23] V. Bareikis and R. Katilius, Noise in Physical Systems and 1/f Fluctuations (World Scientific, Singapore, 1995).

[24] E. W. Bryerton, M. Morgan, and M. W. Pospieszalski, Ultra low noise cryogenic amplifiers for radio astronomy, in Proceedings of the 2013 IEEE Radio and Wireless Symposium (IEEE, Piscataway, NJ, 2013), pp. 358-360.

[25] Sh. Kogan, Electronic Noise and Fluctuations in Solids (Cambridge University Press, Cambridge, 1996).

[26] Herbert B. Callen and Richard F. Greene, On a theorem of irreversible thermodynamics, Phys. Rev. 86, 702 (1952).

[27] H. Nyquist, Thermal agitation of electric charge in conductors, Phys. Rev. 32, 110 (1928).

[28] M. A. Leontovich, Fundamental equations of the kinetic theory of gases from the point of view of the theory of random processes, Zh. Eksp. Teor. Fiz (JETP) 5, 211 (1935).

[29] Gregory H. Wannier, Motion of gaseous ions in a strong electric field. ii, Phys. Rev. 87, 795 (1952).

[30] N. A. Hashitsume, Statistical theory of nonlinear dissipative systems, Prog. Theore. Phys. 15, 369 (1956).

[31] B. B. Kadomtset, Fluctuations in gases, Zh. Eksp. Teor. Fiz. 32, 943 (1957) [Sov. Phys. JETP 5, 771 (1957)].

[32] P. J. Price, Intervalley noise, J. Appl. Phys. 31, 949 (1960).

[33] M. Lax, Fluctuations from the nonequilibrium steady state, Rev. Mod. Phys. 32, 25 (1960).

[34] S. V. Gantsevich, V. L. Gurevich, and R. Katilius, Fluctuations in semiconductors in a strong electric field and the scattering of light by "hot" electrons, Soviet Phys. JETP 30, 276 (1970).

[35] Sh. M. Kogan and A. Ya. Shul'man, Theory of fluctuations in a nonequilibrium electron gas, Soviet Phys. JETP 29, 467 (1969).

[36] M. Lax, Quantum noise. IV. Quantum theory of noise sources, Phys. Rev. 145, 110 (1966).

[37] K. M. van Vliet and J. R. Fasset, Fluctuations due to electronic transitions and transport in solids, in Fluctuation Phenomena in Solids, edited by R. E. Burgess (Academic, New York, 1965), Chap. 7.

[38] Sh. M. Kogan and A. Y. Shul'man, Extraneous random forces and equations for correlation functions in the theory of nonequilibrium fluctuations, Soviet Phys. Solid State 12, 467 (1970).

[39] C. Jacoboni and L. Reggiani, The monte carlo method for the solution of charge transport in semiconductors with applications to covalent materials, Rev. Mod. Phys. 55, 645 (1983).

[40] L. Reggiani, E. Starikov, P. Shiktorov, V. Gruzinskis, and L. Varani, Modelling of small-signal response and electronic noise in semiconductor high-field transport, Semiconductor Science and Technology 12, 141 (1997)

[41] C. J. Stanton and J. W. Wilkins, Nonequilibrium current fluctuations in semiconductors: A boltzmann-equation-green-function approach, Phys. Rev. B 35, 9722 (1987).
[42] C. J. Stanton and John W. Wilkins, Hot-electron noise in twovalley semiconductors: An analytic model, Phys. Rev. B 36, 1686 (1987).

[43] L. Gherardi, A. Pellacani, and C. Jacoboni, Velocity autocorrelation and low temperature mobility of electrons in silicon, Lett. al Nuovo Cimento 14, 225 (1975).

[44] R. Fauquembergue, J. Zimmermann, A. Kaszynski, E. Constant, and Greco Microondes, Diffusion and the power spectral density and correlation function of velocity fluctuation for electrons in si and gaas by monte carlo methods, J. Appl. Phys. 51, 1065 (1980).

[45] D. K. Ferry and J. R. Barker, Generalized diffusion, mobility, and the velocity autocorrelation function for high-field transport in semiconductors, J. Appl. Phys. 52, 818 (1981).

[46] T. Kuhn, L. Reggiani, L. Varani, and V. Mitin, Monte carlo method for the simulation of electronic noise in semiconductors, Phys. Rev. B 42, 5702 (1990).

[47] L. Varani, L. Reggiani, T. Kuhn, T. Gonzalez, and D. Pardo, Microscopic simulation of electronic noise in semiconductor materials and devices, IEEE Transactions on Electron Devices 41, 1916 (1994).

[48] W. Fawcett and H. D. Rees, Calculation of the hot electron diffusion rate for gaas, Phys. Lett. A 29, 578 (1969).

[49] S. Bosi and C. Jacoboni, Monte carlo high-field transport in degenerate GaAs, J. Phys. C: Solid State Phys. 9, 315 (1976).

[50] G. Hill, P. N. Robson, and W. Fawcett, Diffusion and the power spectral density of velocity fluctuations for electrons in inp by monte carlo methods, J. Appl. Phys. 50, 356 (1979).

[51] S. Wang, H. Liu, B. Gao, and H. Cai, Monte carlo calculation of electron diffusion coefficient in wurtzite indium nitride, Appl. Phys. Lett. 100, 142105 (2012).

[52] E. Starikov, P. Shiktorov, V. Gružinskis, L. Reggiani, L. Varani, J C Vaissière, and C. Palermo, Monte carlo calculations of hot-electron transport and diffusion noise in $\mathrm{GaN}$ and $\mathrm{InN}$, Semiconductor Sci. Techn. 20, 279 (2005).

[53] R. Rengel and M. J. Martín, Diffusion coefficient, correlation function, and power spectral density of velocity fluctuations in monolayer graphene, J. Appl. Phys. 114, 143702 (2013).

[54] D. Y. Xing, M. Liu, P. Hu, and C. S. Ting, Diffusion of hot carriers in two-valley semiconductors, J. Phys. C: Solid State Phys. 21, 2881 (1988).

[55] D. Y. Xing, M. Liu, and C. S. Ting, Analytical approach to diffusion of hot carriers in n-type gaas with $\Gamma-1-x$ band structure, Phys. Rev. B 37, 10283 (1988).

[56] I. Iñiguez-de-la Torre, J. Mateos, D. Pardo, and T. González, Monte carlo analysis of noise spectra in self-switching nanodiodes, J. Appl. Phys. 103, 024502 (2008).

[57] H. Rodilla, T. González, D. Pardo, and J. Mateos, High-mobility heterostructures based on inas and insb: A monte carlo study, J. Appl. Phys. 105, 113705 (2009).

[58] S. Karishy, C. Palermo, G. Sabatini, H. Marinchio, L. Varani, J. Mateos, and T. González, Monte carlo calculation of in0.53ga0.47As and InAs noise parameters, in Proceedings of the 2017 International Conference on Noise and Fluctuations (ICNF) (IEEE, Piscataway, NJ, 2017), pp. 1-4.

[59] G. Pizzi, V. Vitale, R. Arita, S. Blügel, F. Freimuth, G. Géranton, M. Gibertini, D. Gresch, C. Johnson, T. Koretsune, J. Ibañez-Azpiroz, H. Lee, Jae-M. Lihm, D. Marchand, A. 
Marrazzo, Y. Mokrousov, J. I. Mustafa, Y. Nohara, Y. Nomura, L. Paulatto, Samuel Poncé, Thomas Ponweiser, J. Qiao, F. Thöle, S. S. Tsirkin, M. Wierzbowska, N. Marzari, D. Vanderbilt, I. Souza, A. A. Mostofi, and J. R. Yates, Wannier90 as a community code: new features and applications, J. Phys.: Condens. Matter 32, 165902 (2020).

[60] A. A. Mostofi, J. R. Yates, Y.-S. Lee, I. Souza, D. Vanderbilt, and N. Marzari, wannier90: A tool for obtaining maximallylocalised wannier functions, Comput. Phys. Commun. 178, 685 (2008).

[61] S. V. Gantsevich, V. L. Gurevich, and R. Katilius, Theory of fluctuations in nonequilibrium electron gas, Rivista Del Nuovo Cimento 2, 1 (1979).

[62] R. H. Fowler, Statistical Mechanics (Cambridge University Press, London, 1936).

[63] P. Giannozzi, S. Baroni, N. Bonini, M. Calandra, R. Car, C. Cavazzoni, D. Ceresoli, G. L. Chiarotti, M. Cococcioni, I. Dabo, A. D. Corso, S. de Gironcoli, S. Fabris, Guido Fratesi, R. Gebauer, U. Gerstmann, C. Gougoussis, A. Kokalj, M. Lazzeri, L. Martin-Samos, N. Marzari, F. Mauri, R. Mazzarello, S. Paolini, A. Pasquarello, L. Paulatto, C. Sbraccia, S. Scandolo, G. Sclauzero, Ari P Seitsonen, A. Smogunov, P. Umari, and R. M. Wentzcovitch, QUANTUM ESPRESSO: A modular and open-source software project for quantum simulations of materials, J. Phys.: Condens. Matter 21, 395502 (2009).

[64] P. Giannozzi, O. Andreussi, T. Brumme, O. Bunau, MB. Nardelli, M. Calandra, R. Car, C. Cavazzoni, D. Ceresoli, M. Cococcioni, N. Colonna, I. Carnimeo, A. Dal Corso, S. de Gironcoli, P. Delugas, R A. DiStasio, A. Ferretti, A. Floris, G. Fratesi, G. Fugallo, R. Gebauer, U. Gerstmann, F. Giustino, T. Gorni, J. Jia, M. Kawamura, H.-Y. Ko, A. Kokalj, E. Küçükbenli, M. Lazzeri, M. Marsili, N. Marzari, F. Mauri, N. L. Nguyen, H.-V. Nguyen, A Otero de-la Roza, L. Paulatto, S. Poncé, D. Rocca, R. Sabatini, B. Santra, M. Schlipf, A. P. Seitsonen, A. Smogunov, I. Timrov, T. Thonhauser, P. Umari, N. Vast, X. Wu, and S. Baroni, Advanced capabilities for materials modeling with quantum ESPRESSO, J. Phys.: Condens. Matter 29, 465901 (2017).

[65] J. Zhou, J. Park, I. Lu, I. Maliyov, X. Tong, and M. Bernardi, Perturbo: A software package for ab initio electronphonon interactions, charge transport and ultrafast dynamics, arXiv:2002.02045 [cond-mat.mtrl-sci] (2020).

[66] J. Sjakste, N. Vast, M. Calandra, and F. Mauri, Wannier interpolation of the electron-phonon matrix elements in polar semiconductors: Polar-optical coupling in gaas, Phys. Rev. B 92, 054307 (2015).

[67] P. Virtanen, R. Gommers, T. E. Oliphant, M. Haberland, Tyler Reddy, D. Cournapeau, E. Burovski, P. Peterson, Warren Weckesser, J. Bright, S. J. van der Walt, Matthew Brett, J. Wilson, K. J. Millman, N. Mayorov, Andrew R. J. Nelson, E. Jones, R. Kern, E. Larson, C. J. Carey, İ. Polat, Yu. Feng, E. W. Moore, J. Vand erPlas, D. Laxalde, J. Perktold, R. Cimrman, I. Henriksen, E. A. Quintero, C. R. Harris, A. M. Archibald, A. H. Ribeiro, F. Pedregosa, P. van Mulbregt, and SciPy 1. 0 Contributors, SciPy 1.0: Fundamental algorithms for scientific computing in Python, Nature Methods 17, 261 (2020).

[68] V. A. Jhalani, J.-J. Zhou, J. Park, C. E. Dreyer, and M. Bernardi, Piezoelectric Electron-Phonon Interaction from Ab Initio Dy- namical Quadrupoles: Impact on Charge Transport in Wurtzite Gan, Phys. Rev. Lett. 125, 136602 (2020).

[69] G. Brunin, H. P. C. Miranda, M. Giantomassi, Miquel Royo, M. Stengel, Matthieu J. Verstraete, X. Gonze, Gian-Marco Rignanese, and G. Hautier, Electron-phonon beyond fröhlich: Dynamical quadrupoles in polar and covalent solids, Phys. Rev. Lett. 125, 136601 (2020).

[70] V. Bareikis, K. Kibickas, J. Liberis, A. Matulionis, R. Miliušyte, J. Paršeliūnas, J. Požela, and P. Sakalas, Velocity overshoot and suppression of diffusivity and microwave noise in short n+-n-n+ structures of gaas, in High-Speed Electronics, edited by Bengt Källbäck and H. Beneking (Springer-Verlag, Berlin, Heidelberg, 1986), pp. 28-31.

[71] J. G. Ruch and G. S. Kino, Transport properties of gaas, Phys. Rev. 174, 921 (1968).

[72] S. Teitel and J. W. Wilkins, Small-signal ac conductivity and velocity overshoot in semiconductor materials, J. Appl. Phys. 53, 5006 (1982).

[73] V. Bareikis, J. Liberis, I. Matulioniene, A. Matulionis, and P. Sakalas, Experiments on hot electron noise in semiconductor materials for high-speed devices, IEEE Transactions on Electron Devices 41, 2050 (1994).

[74] V. Bareikis, V. Viktoravicius, A. Galdikas, and R. Miliusyte, Microwave noise and constant of the coupling between the valleys gamma and 1 in a three-valley model of gaas, Soviet Physics-Semiconductors 14, 847 (1980).

[75] E. M. Conwell and M. O. Vassell, High-field transport in- type GaAs, Phys. Rev. 166, 797 (1968).

[76] B. J. Davydov, On the theory of electron motion in gases and semiconductors, Zh. Eksp. Teor. Fiz. 8, 1069 (1937).

[77] H. R. Skullerud, Longitudinal diffusion of electrons in electrostatic fields in gases, J. Phys. B: Atomic Molecular Phys. 2, 696 (1969).

[78] V. L. Gurevich and R. Katilius, Contribution to the theory of hot electrons in an anisotropic semiconductor, Soviet Phys. JETP 22, 796 (1966).

[79] W. A. Schlup, Definition of noise temperatures $\theta$ in three dimensions and the conjecture $\theta \geqslant t$ in nonequilibrium systems, Physica 69, 485 (1973).

[80] V. Aninkevičius, V. Bareikis, J. Liberis, A. Matulionis, and P. Sakalas, Comparative analysis of microwave noise in gaas and algaas/gaas channels, Solid-State Electronics 36, 1339 (1993).

[81] L. Chaput, Direct solution to the linearized phonon boltzmann equation, Phys. Rev. Lett. 110, 265506 (2013).

[82] P. J. Price, Fluctuations of hot electrons in semiconductors, in Fluctuation Phenomena in Solids, edited by R. E. Burgess (Academic, New York, 1965), Chap. 8.

[83] R. I. Rabinovich, On galvanomagnetic phenomena under hotelectron energy scattering on optical phonons, Soviet Physics Semiconductors 3, 839 (1969).

[84] H. Rodilla, J. Schleeh, P. Nilsson, N. Wadefalk, J. Mateos, and J. Grahn, Cryogenic performance of low-noise inp hemts: A monte carlo study, IEEE Transactions on Electron Devices 60, 1625 (2013).

[85] J. Mateos, H. Rodilla, B. G. Vasallo, and T. Gonzalez, Monte carlo mmodelling of noise in advanced iii-v hemts, J. Comp. Electron. 14, 72 (2015).

[86] L. Lindsay, C. Hua, X. L. Ruan, and S. Lee, Survey of ab initio phonon thermal transport, Materials Today Phys. 7, 106 (2018). 